\title{
Variables influyentes en el miedo y el rendimiento infantil en Ceuta
}

\author{
Influence Variables over Fear and Performance in Children in Ceuta \\ Variáveis influentes do medo e do desempenho infantil em Ceuta
}

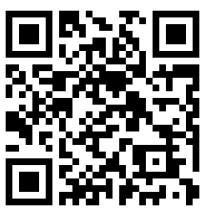

Federico Pulido-Acosta'

Universidad de Granada

Granada, España

feanor_fede@hotmail.com

http://orcid.org/0000-0001-7892-6696

Francisco Herrera-Clavero ${ }^{2}$

Universidad de Granada

Granada, España

fherrera@ugr.es

(iD http://orcid.org/0000-0002-6888-8769

Recibido • Received • Recebido: 05 / 07 / 2016

Corregido • Revised • Revisado: 07 / 11 / 2017

Aceptado • Accepted • Aprovado: 07 / 12 / 2017

\begin{abstract}
Resumen: Existe una enorme preocupación por el bajo rendimiento en las diferentes áreas académicas, lo que promueve la búsqueda de nuevas fórmulas de actuación docente. Estos rápidos cambios hacen que el alumnado deba aprender nuevas habilidades y capacidades con el fin de amoldarse a este proceso en continua evolución. Desde esta perspectiva, centrados en los escasos logros académicos, se pone de relieve la enorme importancia que pueden llegar a tener las emociones y su adecuado control para la mejora de este. El objetivo del presente trabajo fue conocer los predictores del miedo y el rendimiento académico de estudiantes, de 6 a 12 años, en la ciudad de Ceuta. Para ello, se ha contado con 404 sujetos participantes, de los cuales, el $47.8 \%$ son niños, el $52.2 \%$ niñas, el $68.8 \%$ son musulmanes y el $31.2 \%$ cristianos. Como instrumentos se han empleado la adaptación de Ascensio, Vila, Robles-García, Páez, Fresán y Vázquez (2012) del Inventario de miedo para niños (FSSCII) y las calificaciones del alumnado. Los resultados reflejan niveles medio-altos en las variables de estudio. El principal predictor del miedo es el género, actuando también como predictores estatus,
\end{abstract}

\footnotetext{
${ }^{1}$ Maestro, Licenciado en Psicopedagogía, Doctor en Psicología por la Universidad de Granada, colaborador en psicología evolutiva y de la educación de la Universidad de Granada, en la Facultad de Educación y Humanidades de Ceuta. Dirección postal: Parques de Ceuta, 2a fase, portal 3, 8 C, CP: 51002, Ceuta. Teléfono: +32664779270.

${ }^{2}$ Maestro, Doctor en Filosofía y Ciencias de la Educación por la UNED, Doctor en Psicología por la Universidad de Granada y profesor titular en psicología evolutiva y de la educación de la Universidad de Granada, destinado en la Facultad de Educación y Humanidades de Ceuta, Dirección postal: Facultad de Educación, Economía y Tecnología de Ceuta, Dpto. de Psicología Evolutiva y de la Educación, Cortadura del Valle, s/n, CP: 51001, Ceuta. Teléfono: + 32649498851.
} 
doi: http://dx.doi.org/10.15359/ree.22-1.15

URL: http://www.una.ac.cr/educare

CORREO: educare@una.cr

rendimiento, cultura y edad. Los predictores del rendimiento son la cultura, el miedo, el estatus y el género, la edad aparece en tres factores. Se halló relación significativa entre miedo y rendimiento, ambos inversamente proporcionales. Todo ello nos lleva a considerar y potenciar las competencias emocionales como una medida necesaria para mejorar el proceso de aprendizaje del alumnado, situación que tanto se demanda a nuestro sistema educativo.

Palabras claves: Emoción; miedo; rendimiento académico; contexto pluricultural.

Abstract: The low performance in different academic areas causes an enormous preoccupation; this situation promotes the search of new formulas for teaching interventions. These fast changes cause that students must learn new abilities and capacities that let them conform to this process in continuous evolution. From this perspective, with preoccupation for the little academic achievements, the emphasis is on the enormous importance that the emotions and their suitable control for the improvement of itself could have. The objective was to reflect on the predictors of fear and the academic achievement, and the influence over each other, in children aged 6 to 12 from Ceuta City. To do so, we had 404 participants: $47.8 \%$ were boys, 52.2\% girls; $68.8 \%$ were Muslims, and 31.2\% Christians. As tools, we implemented the Fear Survey Schedule for Children and AdolescentsII (FSSC-II), the version that Ascensio et al. (2012) adapted to the Spanish language; we also used the children's grades. The results showed medium-high levels on all of the research variables. The first predictor of fear was gender. Other predictors were status, performance, culture, and age. The predictors of performance were culture, fear, status, and gender. Age worked as a predictor in three factors. We have found a significant relationship between fear and performance. All this leads to consider and increase the emotional competencies as a necessary measure to improve the students' process of learning, a situation needed in our education system.

Keywords: Emotion; fear, academic performance; multicultural context.

Resumo: Existe uma grande preocupação pelo baixo desempenho nas diferentes áreas acadêmicas. Isto promove a busca de novas formas de desempenho docente. Estas mudanças rápidas fazem com que os alunos devam aprender novas habilidades e capacidades com a finalidade de adaptar-se a este processo em continua evolução. A partir desta perspectiva, e tendo presente os escassos resultados acadêmicos, salienta-se a enorme importância que podem ter as emoções e seu adequado controle para a melhora deste desempenho. O objetivo deste estudo foi conhecer o precedente de medo e desempenho acadêmico dos alunos de 6 a 12 anos na cidade de Ceuta. Para este fim participaram 404 crianças, dos quais $47,8 \%$ são meninos e $52,2 \%$ meninas, $68,8 \%$ muçulmanos e $31,2 \%$ cristãos. Foram usados como instrumentos, a adaptação de Ascensio (2012) do Inventário do medo para crianças (FSSC-II) e as notas dos alunos. Os resultados refletem níveis médio-alto nas variáveis do estudo. O principal precedente de medo é o gênero, também o status, o desempenho, a cultura e a idade. Os precedentes de desempenho são a cultura, o medo, status e o gênero, a idade aparece em três fatores. Se encontrou uma relação significativa entre o medo e o desempenho, os dois inversamente proporcionais. Tudo isso nos leva a considerar e valorizar as competências emocionais como uma medida necessária para melhorar o processo de aprendizagem dos alunos, uma situação muito demandada pelo nosso sistema educativo.

Palavras-chave: Emoção; medo; desempenho acadêmico; contexto pluricultural. 


\section{Introducción}

En la actualidad, dentro del ámbito educativo, aparecen problemas ante los que los grupos profesionales de la educación no saben contestar de forma efectiva. Muchas de las soluciones a estos problemas no son otras que la inclusión del mundo de las emociones dentro del currículo. Solo una intervención suficientemente profunda podrá tener éxito en este sentido, lo que sirve para seguir demandando la incorporación del mundo emocional en el currículo de una forma verdadera y eficaz. Sin embargo, la intervención en este ámbito plantea importantes problemas que solo pueden ser resueltos con amplias consideraciones formativas, de planificación y evaluativas dentro del profesorado. Un desarrollo inadecuado de este tipo de habilidades puede influir de forma negativa en el alumnado. Esto puede ocurrir tanto dentro como fuera del ámbito educativo, y hace que aparezcan múltiples dificultades: estados emocionales negativos, problemas de estrés y ansiedad, dificultades para interactuar con otras personas, conductas desajustadas... (Gutiérrez y Expósito, 2015).

Los maestros y las maestras que trabajan de forma adecuada las habilidades socioemocionales hacen que sus estudiantes muestren una conducta exploratoria más intensa durante las etapas iniciales del proceso educativo. Dicha conducta, durante estos periodos educativos, aparece como una necesidad básica asociada al adecuado desarrollo cognitivo y emocional, lo que permite una experiencia educativa más profunda y con efectos positivos a largo plazo (Pascal y Bertram, 1997).

Centrados en intereses personales, se ha optado por el estudio de las emociones de carácter negativo, y se ha encontrado en el miedo el principal punto de mira. Los niños y las niñas temen a situaciones muy variadas que, en parte, dependen de la edad, uno de los factores que influyen en la intensidad y el número de miedos. A medida que el sujeto se va desarrollando emocionalmente, desciende su manifestación de miedo, algo que aparece en trabajos (Kushnir, Gothelf y Sadeh, 2014; Pulido y Herrera, 2015) que lo consideran propio del proceso evolutivo del sujeto. Otra línea de estudios se mueve sobre la incidencia del género sobre el desempeño emocional (Miloyan, Bulley, Pachana y Byrne, 2014; Pinto, Dutra, Filgueiras, Juruena y Stingel, 2013; Pulido y Herrera, 2015). De esta manera, el género se muestra como variable verdaderamente importante en la incidencia e intensidad de los miedos. También se puede contemplar la enorme incidencia que tiene el entorno sobre el miedo, donde se observa la influencia de los acontecimientos externos en el desarrollo emocional. El proceso de socialización llevado a cabo por familias es el punto de partida de las diferencias encontradas, si se considera el grupo cultural de procedencia (Soriano y González, 2013). La pertenencia a un grupo cultural es, por tanto, un elemento a tener en cuenta, los individuos pertenecientes a minorías culturales son quienes presentan manifestaciones superiores en emociones negativas (Merchán, Bermejo y González, 2014; Pulido y Herrera, 2015). 
doi: http://dx.doi.org/10.15359/ree.22-1.15

URL: http://www.una.ac.cr/educare

CORREO: educare@una.cr

El trabajo se enfoca sobre el ámbito académico, del que se toma la variable rendimiento, entendido como nivel de conocimientos y destrezas escolares exhibidos por el estudiantado. Este tipo de habilidades emocionales está íntimamente relacionado con el desempeño personal, social, académico y profesional del alumnado, independientemente de su edad, género o cultura. Ante esta realidad, se demanda la necesidad de incorporar este tipo de habilidades en el currículo (Gutiérrez y Expósito, 2015). Para el rendimiento se consideraron las calificaciones escolares como su indicativo.

Pare ello, Gómez-Castro (1986, p. 269) justifica:

Al comparar los resultados de las calificaciones objetivas a través de una prueba estandarizada y las que otorgan los profesores, indica cierta asociación entre ellas. Todas las correlaciones obtenidas entre las distintas subpruebas y las calificaciones son estadísticamente significativas ( $p>01)$.

Si se consideran los trabajos de Pulido y Herrera (2015) y Balongo y Mérida (2016), la diversidad cultural es un factor por tener en cuenta, dado que se registran resultados superiores entre los sujetos pertenecientes a la cultura "predominante".

Si se hace referencia a la interacción entre las dos principales variables de estudio, se debe mencionar la existencia de una importante relación entre la inteligencia emocional, la ansiedad y las emociones básicas de carácter negativo. Del mismo modo, estos estados emocionales se relacionan con elementos como la satisfacción con la vida, la salud mental (Extremera, Durán y Rey, 2009) y la satisfacción laboral (Pena, Rey y Extremera, 2012). La mayoría o incluso todos estos factores repercuten de manera clara sobre el desarrollo educativo.

Tras justificar la relación de las emociones de carácter negativo con múltiples áreas de la vida, se pretende contemplar si estas pudieran incidir en el desarrollo académico, dentro del ámbito educativo. El presente estudio tiene como propósito central identificar cuáles son los principales predictores de miedo y rendimiento académico. Como posibles variables predictoras se consideró el género del sujeto, su grupo cultural de pertenencia, su curso (edad) y su nivel socioeconómico y cultural. En segundo lugar, se pretende estudiar cuál es la relación, si es que existe, entre las variables de estudio. Para responder a estos objetivos, se llevó a cabo una recogida de información en alumnado perteneciente a los cursos desde $1^{\circ}$ hasta $6^{\circ}$ de la etapa de Educación Primaria, de cuatro centros educativos de Ceuta, una ciudad española situada en el norte de África. Esta ciudad es especialmente indicada, dado que conviven dos grupos culturales diferentes. Como característica fundamental, de importancia para vincular el grupo cultural con la religión e incluso con la lengua materna, la población árabe musulmana presenta una procedencia marroquí con un altísimo nivel de analfabetismo, así como un estatus socioeconómico y cultural bajo, una lengua materna diferente al castellano y una marcada presencia de su religión, el islam, en sus vidas (Herrera, 2000). Teniendo en cuenta la revisión bibliográfica, se espera que el género, 
la edad, la cultura y el estatus funcionarán como predictores para la variable miedo. Para el caso del rendimiento no se esperan diferencias en este sentido, salvo en el caso de la cultura. Se entiende que esta será uno de los predictores más importantes. Finalmente, se esperan relaciones entre miedo y rendimiento, que actúan en sentido contrario.

\section{Metodología}

\section{Participantes}

Para afrontar este estudio se seleccionó una muestra integrada por 404 participantes que reflejan las características del contexto pluricultural. En función de la variable género, se dan porcentajes bastante equilibrados entre sí, siendo el $47.8 \%$ niños y el resto de la muestra (52.2\%) chicas. Considerando la cultura, el grupo mayoritario es el de participantes pertenecientes a la cultura-religión musulmana, que constituyen el $68.8 \%$ de la muestra, mientras que el 31.2\% son cristianos. El método para la selección de la muestra fue aleatorio de sujetos disponibles. De esta manera, después de solicitar permiso por escrito, tanto a la Administración Educativa, como a la Dirección de los centros y, por supuesto, a los padres y madres de familia, se procedió a la recogida de la información, contestaron quienes lo desearon. Se tomó en cuenta un error muestral del $3 \%$.

\section{Instrumentos}

La evaluación de la variable miedo se llevó a cabo a través de la versión del Inventario de miedo para niños (FSSC-II) de Ascensio et al. (2012). Se realizaron las pruebas psicométricas para conocer su consistencia interna, lo que dio un valor más que aceptable ( $\alpha=.955)$. Posteriormente, se empleó un análisis factorial confirmatorio (AFC), con el modelo de máxima verosimilitud, con rotación varimax. El primero de los cinco factores que se vinculó con miedos relacionados con lo desconocido y con elementos extraños representa el $23.904 \%$ de la varianza explicada. El segundo de los factores representa el $10.254 \%$ de la varianza explicada y hace referencia a los miedos relacionados con la muerte (Miedo a la muerte). El tercer factor, hace referencia al miedo provocado por animales que representa el 3.992\% de la varianza explicada. El miedo a la crítica y el engaño es la categoría que se corresponde con el cuarto factor, que representan el 3.058\% de la varianza explicada. El quinto y último factor, relacionado con miedos escolares representa el $2.552 \%$ de la varianza explicada. Entre todos los factores suman una varianza explicada total del $43.760 \%$, con un total de 78 reactivos.

Para la medida del rendimiento se tomaron las calificaciones que el profesorado, en su proceso de evaluación, otorga a cada uno de sus estudiantes. Esta medida resulta válida si se tiene en cuenta el, ya mencionado, trabajo de Gómez-Castro (1986). 
doi: http://dx.doi.org/10.15359/ree.22-1.15

URL: http://www.una.ac.cr/educare

CORREO: educare@una.cr

\section{Procedimiento}

Para poder cumplir los objetivos se empleó el método no experimental ex post facto. Una vez recogida la información, que se llevó a cabo dentro de cada una de las aulas de los centros que participaron en la experiencia, en presencia del profesor o profesora de cada curso, se comenzó a construir la base de datos. Para ello, se empleó el programa de análisis estadístico SPSS (2009). Se comenzó llevando a cabo un análisis descriptivo. Primero se hizo de una forma general, para pasar, posteriormente, a un estudio comparativo, que consideró como variables independientes el género, el curso (edad), el grupo cultural y el estatus socioeconómico y cultural. En el apartado inferencial, se usó el análisis de regresión, empleando el método de "pasos sucesivos". El propósito de este análisis fue predecir qué variable explica, en mayor medida, por un lado, el miedo y, por otro, el rendimiento académico. También se emplearon como variables criterio cada una de las dimensiones que forman parte del miedo y cada una de las materias, cuyas medias constituyen el rendimiento general. Como variables predictoras se usaron las mismas descritas para el estudio comparativo (género, edad, cultural y estrato socioeconómico) añadiéndoles el miedo o el rendimiento (siempre que no actuaran como variable criterio). De esta manera, se han podido determinar las dimensiones que mejor predicen cada una de las variables criterio. Del mismo modo, se pueden conocer las posibles relaciones entre miedo y rendimiento académico. así como la interacción entre las dos variables de estudio.

\section{Resultados}

Se inicia este apartado con el análisis descriptivo general, comenzando por la variable miedo. Las puntuaciones se alejan de ambos extremos, acercándose más a los niveles superiores, siendo la opción de bastante miedo la más alcanzada.

Los resultados resumidos en la Tabla 1 muestran que el miedo total está bajo la influencia de las variables estatus, género, curso/edad y rendimiento. El porcentaje de varianza total es de $26.2 \%\left(R^{2}=.262\right)$. El mayor poder determinante lo ejerce el género, que explica el $28.3 \%$ de la varianza total $(\beta=.283)$. El valor que el género tiene en la función es positivo, lo que indica que son las chicas las que tienen puntuaciones más altas (debido a que se asoció el valor 1 con los varones y el 2 con las mujeres). El resto de variables alcanzan valores negativos, lo que indica niveles más altos de miedo entre los niños de menor edad, los que ocupan un estatus más bajo y los que obtienen peores calificaciones. Todo ello se puede observar en la siguiente función de regresión (Tabla 1 ). 
Tabla 1: Regresión miedo total

\begin{tabular}{lcccc}
\hline & $\mathrm{R}=.512$ & $\mathrm{R}^{2}=.262$ & $\mathrm{~F}=33.222$ & $\mathrm{p}=.0000$ \\
\multicolumn{1}{c}{ Variables } & $\mathrm{B}$ & $\beta$ & $\mathrm{t}$ & $\mathrm{p}$ \\
\hline Constante general & 3.280 & & 16.566 & .0000 \\
Estatus & -.183 & -.269 & -5.604 & .000 \\
Género & .454 & .283 & 6.336 & .000 \\
Curso/Edad & -.128 & -.228 & -4.761 & .000 \\
Rendimiento total & -.012 & -.133 & -2.958 & .003 \\
\hline
\end{tabular}

Miedo total= $3.280-.183$ (estatus) +.454 (género) -.128 (curso/edad) -.012 (rendimiento total).

Los resultados de la regresión múltiple(Tabla 2) indican que el factor de miedo a los animales y al peligro está bajo la influencia de la variable género y también del estatus. El conjunto de ellas da cuenta del $15.7 \%$ de la varianza en este factor $\left(R^{2}=.157\right)$. El mayor poder determinante lo tiene el género, que explica el $30.7 \%$ de la varianza ( $\beta=.307)$. Los valores positivos en el género indican mayores puntuaciones entre las chicas, mientras que el estatus indica que son los que tienen un nivel socioeconómico inferior los que evidencian niveles superiores.

Tabla 2: Regresión miedo animales

\begin{tabular}{lcccc}
\hline \multicolumn{1}{c}{$\mathrm{R}=.397$} & $\mathrm{R}^{2}=.157$ & $\mathrm{~F}=35.164$ & $\mathrm{p}=.0000$ & \\
\hline \multicolumn{1}{c}{ Variables } & $\mathrm{B}$ & $\beta$ & $\mathrm{t}$ & $\mathrm{P}$ \\
\hline Constante general & 2.387 & & 13.251 & .0000 \\
Género & .627 & .307 & 6.496 & .000 \\
Estatus & -.216 & -.249 & -5.275 & .000 \\
\hline
\end{tabular}

Miedo animales $=2.387+.627$ (género) -.216 (estatus).

La regresión arroja los datos (Tabla 3) que permiten ver como el miedo a la muerte está bajo la influencia de las variables estatus y género. El conjunto de ellas da cuenta del $6.5 \%$ de la varianza en este factor de miedo $\left(R^{2}=.065\right)$. Ambas variables son igualmente determinantes, ya que tanto género como estatus explican el $18 \%$ de la varianza $(\beta=.18)$. Como ocurría en los casos anteriores, el valor de la variable género en la función vuelve a ser positivo, lo que indica que son las participantes femeninas las que tienen puntuaciones más altas. El estatus es inversamente proporcional al miedo a la muerte. 
doi: http://dx.doi.org/10.15359/ree.22-1.15

URL: http://www.una.ac.cr/educare

CORREO: educare@una.cr

Tabla 3: Regresión miedo muerte

\begin{tabular}{lccccc}
\hline & $\mathrm{R}=.255$ & $\mathrm{R}^{2}=.065$ & $\mathrm{~F}=13.069$ & $\mathrm{p}=.0000$ & \\
\hline \multicolumn{1}{c}{ Variables } & $\mathrm{B}$ & $\beta$ & $\mathrm{t}$ & $\mathrm{P}$ \\
\hline Constante general & 3.223 & & 20.912 & .0000 \\
Estatus & -.126 & -.180 & -3.608 & .000 \\
Género & .298 & .180 & 3.607 & .000 \\
\hline
\end{tabular}

Miedo muerte $=3.22-.126$ (estatus) +.298 (género) .

En la Tabla 4 se reflejan los resultados que demuestran que el miedo a lo desconocido está bajo la influencia de las variables curso/edad, estatus, género y rendimiento, que dan cuenta del $34.3 \%$ de la varianza $\left(R^{2}=.343\right)$. En este caso, es la edad la variable que ejerce el mayor poder determinante que por sí sola explica el $36.9 \%$ de la varianza total $(\beta=.369)$. Los varones vuelven a presentar puntuaciones inferiores en este factor, de la misma manera que los sujetos mayores y los pertenecientes a grupos socioculturales más elevados. Los que mejores calificaciones obtienen, también, son los que menos niveles de miedo presentan.

Tabla 4: Regresión miedo desconocido

\begin{tabular}{lcccc}
\hline & $\mathrm{R}=.586$ & $\mathrm{R}^{2}=.343$ & $\mathrm{~F}=48.945$ & $\mathrm{p}=.0000$ \\
\multicolumn{1}{c}{ Variables } & $\mathrm{B}$ & $\beta$ & $\mathrm{t}$ & $\mathrm{P}$ \\
\hline Constante general & 3.647 & & 17.276 & .0000 \\
Curso/Edad & -.235 & -.369 & -8.178 & .000 \\
Estatus & -.178 & -.231 & -5.110 & .000 \\
Género & .423 & .233 & 5.537 & .000 \\
Rendimiento total & -.022 & -.212 & -5.005 & .000 \\
\hline
\end{tabular}

Miedo desconocido $=3.647-.235$ (curso/edad) -.178 (estatus) +.423 (género) -.022 (rendimiento total).

Los resultados de la regresión (Tabla 5) permiten predecir la puntuación del factor miedos escolares, conociendo la cultura/religión, el rendimiento total y el género del sujeto. Esto nos da una varianza del $11.1 \%$ en este factor $\left(R^{2}=.111\right)$. El rendimiento, con el mayor poder determinante, explica el $20.9 \%$ de la varianza total $(\beta=.209)$. De nuevo, son las chicas las que evidencian resultados superiores. Lo mismo ocurre con los musulmanes y con los que tienen un rendimiento más bajo. Todo se puede ver, si se sigue la función que aparece a continuación.

8 Federico Pulido-Acosta y Francisco Herrera-Clavero

Los artículos de la Revista Electrónica Educare del Centro de Investigación y Docencia en Educación de la Universidad Nacional, Costa Rica, se comparten bajo términos de la Licencia Creative Commons: Reconocimiento, № Comercial, Sin 0bra Derivada 3.0 Costa Rica. Las autorizaciones adicionales a las aquí delimitadas se pueden obtener en el correo: educare@una.cr 
Tabla 5: Regresión miedos escolares

\begin{tabular}{lcccc}
\hline & $\mathrm{R}=.333$ & $\mathrm{R}^{2}=.111$ & $\mathrm{~F}=15.863$ & $\mathrm{p}=.0000$ \\
\hline \multicolumn{1}{c}{ Variables } & $\mathrm{B}$ & $\beta$ & $\mathrm{t}$ & $\mathrm{P}$ \\
\hline Constante general & 2.145 & & 7.376 & .000 \\
Cultura/Religión & .383 & .191 & 3.828 & .000 \\
Rendimiento total & -.022 & -.209 & -4.158 & .000 \\
Género & .255 & .140 & 2.854 & .005 \\
\hline
\end{tabular}

Miedos escolares $=2.145+.383$ (cultura/religión) -.022 (rendimiento total) ++.255 (género).

Para el factor miedo al engaño y la crítica, los cálculos de regresión reflejan que se puede anticipar, si se conoce la variable género. Esta representa el $7.5 \%$ de la varianza en miedos médicos $\left(R^{2}=.075\right)$, y explica un $27.4 \%$ de esta varianza total $(\beta=.274)$. Las chicas vuelven a tener niveles superiores. Los datos que han permitido realizar esta descripción aparecen resumidos en la Tabla 6.

Tabla 6: Regresión miedo crítica

\begin{tabular}{cccccc}
\hline & $\mathrm{R}=.274$ & $\mathrm{R}^{2}=.075$ & $\mathrm{~F}=30.705$ & $\mathrm{p}=.0000$ & \\
\hline Variables & $\mathrm{B}$ & $\beta$ & $\mathrm{t}$ & $\mathrm{P}$ \\
\hline Constante general & 1.380 & & 10.881 & .000 \\
Género & .439 & .274 & 5.541 & .000 \\
\hline
\end{tabular}

Miedos engaño $=1.380+.439$ (género).

La variable predictora más importante (aparece en los totales y en todos los factores) es el género. Las niñas tienen un nivel más alto de miedo que los niños. Los resultados reflejan que estas (media de 140.38) manifiestan niveles superiores en comparación con los niños (118.27). Los resultados de la prueba ANOVA ponen de manifiesto que las variables relacionadas con el miedo se encuentran bajo la influencia del género. Aparecen diferencias significativas en miedo total $(p=.000)$, a lo desconocido $(p=.000)$, a la muerte $(p=.001)$, a los animales $(p=.000)$, al engaño y a la crítica $(p=.000)$ y los miedos escolares $(p=.001)$. Las niñas reflejan niveles más altos en todos los casos.

La variable estatus actúa como predictor, tanto en los totales como en tres de los cinco factores que forman parte del miedo. Se observa un descenso progresivo en los niveles de miedo, a medida que aumenta el estatus. El hecho de pertenecer a un determinado estatus influye en el miedo total $(p=.000)$, a lo desconocido $(p=.000)$, a la muerte $(p=.004)$, a los animales $(p=.000)$ y los miedos escolares $(p=.000)$. No se obtuvieron en el miedo al engaño y a la crítica ( $p=.328$; en esta variable no actúa como predictor). 
doi: http://dx.doi.org/10.15359/ree.22-1.15

URL: http://www.una.ac.cr/educare

CORREO: educare@una.cr

Actuando como predictor en los totales y en un factor, aparece la edad. Los niños o niñas de menor edad registran niveles más altos (su media es 201.14) de miedo total. El último nivel de primaria (121.8) es en el que se registran resultados más bajos de miedo. Estas diferencias resultan significativas para el miedo total $(p=.000)$, el miedo a lo desconocido $(p=.000)$, el miedo a la muerte $(p=.000)$, el miedo a los animales $(p=.000)$, el miedo al engaño y a la crítica $(p=.000)$ y los miedos escolares $(p=.000)$.

Finalmente, entre las variables sociodemográficas predictoras, actuando en un solo factor, aparece la cultura. Considerando la cultura, el alumnado musulmán manifiesta niveles más altos (con una media de 136.41) de miedo que los cristianos (113.48). La cultura no solo influye en los miedos escolares $(p=.000)$, sino en el miedo total $(p=.000)$, a lo desconocido $(p=.000)$, a la muerte $(p=.039)$ y a los animales ( $p=.002)$. No fue así para el miedo al engaño y a la crítica $(p=.952)$.

Para el rendimiento académico, expresado por la nota media, el resultado más habitual (30.6\%) es el notable. La siguiente calificación es el "bien" (21\%). A continuación, aparecen, muy próximas entre sí, las calificaciones de suspenso (con un 19.8\%) y aprobado (19.2\%). La calificación con porcentajes más bajos (9.4\%) es el sobresaliente.

Los resultados resumidos en la Tabla 7 muestran que el rendimiento académico está bajo la influencia de cultura/religión, género, miedo total y estatus. El conjunto da cuenta del $8.4 \%$ de la varianza en el rendimiento $\left(R^{2}=.084\right)$. El mayor poder determinante lo ejerce la variable cultura/religión, que por sí sola explica el $30.3 \%$ de la varianza en rendimiento $(\beta=.303)$. Existe una relación inversamente proporcional entre el rendimiento académico y la cultura/religión, así como el miedo total y el estatus. La cultura/religión adquiere un valor negativo, lo que indica que los grupos participantes cristianos obtienen resultados superiores en rendimiento académico. Cuanto más alto es el nivel socioeconómico y las puntuaciones de miedo total, más bajas son las calificaciones obtenidas por la persona. El género adopta valores positivos, indicando que son las niñas las que obtienen resultados superiores (asociadas al valor 2).

Tabla 7: Regresión rendimiento académico total

\begin{tabular}{lcccc}
\hline & $\mathrm{R}=.290$ & $\mathrm{R}^{2}=.084$ & $\mathrm{~F}=8.563$ & $\mathrm{p}=.000$ \\
\multicolumn{1}{c}{ Variables } & $\mathrm{B}$ & $\beta$ & $\mathrm{t}$ & $\mathrm{p}$ \\
\hline Constante general & 4.792 & & 8.251 & .000 \\
Cultura/Religión & -.871 & -.303 & -4.225 & .000 \\
Género & .418 & .160 & 3.101 & .002 \\
Miedo total & -.243 & -.149 & -2.694 & .007 \\
Estatus & -.205 & -.185 & -2.489 & -.013 \\
\hline
\end{tabular}

Rendimiento académico total= $4.792-.871$ (cultura/religión) +.418 (género) - .243 (miedo total) - .205 (estatus). 
El rendimiento en la materia de Lengua Castellana (Tabla 8) está también bajo la influencia de las variables cultura/religión, curso/edad, género y miedo total. Entre todas ofrecen un $9.9 \%$ de la varianza en rendimiento en esta materia $\left(R^{2}=.099\right)$. El mayor poder determinante lo ejerce la variable cultura, que por sí sola explica el $23.2 \%$ de esta varianza $(\beta=.232)$. Todas las variables, excepto el género, son inversamente proporcionales. Esto indica mayores niveles entre los grupos cristianos, los menores (menos edad) y los que reflejan niveles inferiores de miedo. La única variable con valores positivos, en la función de regresión, es el género, que indica resultados superiores para las chicas.

Tabla 8: Regresión rendimiento Lengua Castellana

\begin{tabular}{lcccc}
\hline & $\mathrm{R}=.314$ & $\mathrm{R}^{2}=.099$ & $\mathrm{~F}=10.284$ & $\mathrm{p}=.000$ \\
\multicolumn{1}{c}{ Variables } & $\mathrm{B}$ & $\beta$ & $\mathrm{t}$ & $\mathrm{P}$ \\
\hline Constante general & 4.947 & & 10.840 & .000 \\
Cultura/Religión & -.695 & -.232 & -4.477 & .000 \\
Curso/Edad & -.170 & -.178 & -3.374 & .001 \\
Género & .401 & .147 & 2.873 & .004 \\
\hline Miedo total & -.256 & -.150 & -2.717 & .007 \\
\hline
\end{tabular}

Rendimiento Lengua Castellana $=4.947-.695$ (cultura/religión) -.170 (curso/edad) +.401 (género) - .256 (miedo total).

La regresión arroja los datos (Tabla 9) que permiten ver como el rendimiento en Matemáticas, está bajo la influencia de las variables cultura/religión y edad. El conjunto de ambas da cuenta del $5.7 \%$ de la varianza en la IE ( $\left.R^{2}=.057\right)$. La variable más determinante resulta, de nuevo, la cultura/ religión ( $\beta=.236)$, que por sí sola explica el $23.6 \%$ de la varianza en rendimiento en la asignatura Matemáticas. El valor que adopta la variable cultura/religión es negativo, lo que indica que son los grupos musulmanes (asociados a un valor numérico más alto) los que obtienen resultados inferiores en esta materia. Ocurre lo mismo para los que son mayores.

Tabla 9: Regresión rendimiento Matemáticas

\begin{tabular}{lccccc}
\hline & $\mathrm{R}=.239$ & $\mathrm{R}^{2}=.057$ & $\mathrm{~F}=11.430$ & $\mathrm{p}=.000$ & \\
\hline \multicolumn{1}{c}{ Variables } & $\mathrm{B}$ & $\beta$ & $\mathrm{t}$ & $\mathrm{P}$ \\
\hline Constante general & 4.674 & & 12.380 & .0000 \\
Cultura/Religión & -.709 & -.236 & -4.593 & .000 \\
Curso/Edad & -.116 & -.121 & -2.351 & .019 \\
\hline
\end{tabular}

Rendimiento Matemáticas= $4.674-.709$ (cultura/religión) - .116 (curso/edad) 
doi: http://dx.doi.org/10.15359/ree.22-1.15

URL: http://www.una.ac.cr/educare

CORREO: educare@una.cr

Los resultados de la regresión (Tabla 10) permiten predecir el rendimiento en el área de conocimiento del medio, si se conocen las variables cultura/religión, curso/edad, género y miedo total. Todas ellas suman el $9.2 \%$ de la varianza en el rendimiento en esta materia $\left(R^{2}=.092\right)$. Como ocurre con el resto de las variables relacionadas con el rendimiento, el mayor poder determinante lo ejerce la cultura, que por sí sola explica el $22.7 \%$ de la varianza en esta materia ( $\beta=.227$ ). De nuevo su valor es negativo, lo que indica niveles mayores entre los grupos cristianos. También son negativos los valores de las variables curso y miedo, lo que indica la realidad ya comentada. La variable género alcanza valores positivos.

Tabla 10: Regresión rendimiento conocimiento del medio

\begin{tabular}{|c|c|c|c|c|}
\hline & $\mathrm{R}=.303 \quad \mathrm{R}^{2}=.092$ & $F=9.470$ & $p=.000$ & \\
\hline Variables & B & $\beta$ & $\mathrm{t}$ & $P$ \\
\hline Constante general & 4.882 & & 10.514 & .000 \\
\hline Cultura/Religión & -.690 & -.227 & -4.366 & .000 \\
\hline Curso/Edad & -.155 & -.160 & 3.027 & .003 \\
\hline Género & .423 & .153 & 2.974 & .003 \\
\hline Miedo total & -.231 & -.134 & -2.408 & .017 \\
\hline
\end{tabular}

Para la materia de Inglés, los cálculos de regresión reflejan que el rendimiento se puede anticipar si se conocen las variables miedo total, estatus, género y cultura/religión. Esto da lugar al $5.9 \%$ de la varianza en rendimiento en esta materia $\left(R^{2}=.059\right)$. De esta varianza, el miedo total tiene el mayor poder determinante, y explica el $24.6 \%(\beta=.246)$. Existe una relación inversamente proporcional entre el rendimiento académico en Inglés y el miedo. Lo mismo que ocurre con el estatus y la cultura, al indicar niveles más altos entre quienes reflejan un nivel socioeconómico más bajo y entre los grupos cristianos. De nuevo son las chicas las que reflejan resultados superiores. Los datos que han permitido realizar esta descripción aparecen resumidos en la Tabla 11. 
Tabla 11: Regresión rendimiento Inglés

\begin{tabular}{lccccc}
\hline & $\mathrm{R}=.243$ & $\mathrm{R}^{2}=.059$ & $\mathrm{~F}=5.878$ & $\mathrm{p}=.000$ & \\
\hline \multicolumn{1}{c}{ Variables } & $\mathrm{B}$ & $\beta$ & $\mathrm{t}$ & $\mathrm{P}$ \\
\hline Constante general & 4.974 & & 7.588 & .000 \\
Miedo total & -.411 & -.246 & -4.026 & .000 \\
Estatus & -.301 & -.243 & -3.235 & .001 \\
Género & .351 & .120 & 2.306 & .022 \\
Cultura/Religión & -.510 & -.159 & -2.191 & .026 \\
\hline
\end{tabular}

Rendimiento Inglés= $4.974-.411$ (miedo total) -.301 (estatus) +.351 (género) -

- .510 (cultura/religión).

Para concluir con las asignaturas, los datos reflejados en la Tabla 12 permiten calcular la nota del sujeto en Religión en función de la cultura/religión. En esta ocasión, la cultura es la única variable influyente, siendo inversamente proporcional al rendimiento en esta materia. La varianza total es del $4.6 \%$ de la varianza total $\left(R^{2}=.046\right)$ y explica la única variable predictora, el $21.5 \%$ de la varianza total $(\beta=.215)$.

Tabla 12: Regresión rendimiento Religión

\begin{tabular}{cccccc}
\hline & $\mathrm{R}=.215$ & $\mathrm{R}^{2}=.046$ & $\mathrm{~F}=18.366$ & $\mathrm{p}=.0000$ & \\
\hline Variables & $\mathrm{B}$ & $\beta$ & $\mathrm{t}$ & $\mathrm{P}$ \\
\hline Constante general & 4.428 & & 18.711 & .0000 \\
Cultura/Religión & -.573 & -.215 & -4.286 & .000 \\
\hline
\end{tabular}

Rendimiento Religión= $4.428-.573$ (cultura/religión).

La principal variable predictora, la cultura, funciona como tal en los totales y todos los factores. Las calificaciones son algo más altas entre la población de cultura cristiana. Las medias refuerzan estas diferencias, que favorecen, de manera evidente, al grupo de religión cristiana en el rendimiento global (cristianos 7.17 y musulmanes 6.19). Por eso, la cultura/religión influye en la media de rendimiento total $(p=.000)$, el rendimiento en Lengua Castellana $(p=.000)$, en Matemáticas $(p=.000)$, en Conocimiento del Medio $(p=.000)$ y en Religión ( $p=.000)$. El rendimiento en Inglés $(p=.219)$ supone la única materia en la que no se obtienen diferencias significativas. En todos los casos, el grupo de cristianos obtienen mejores resultados que el de musulmanes. 
doi: http://dx.doi.org/10.15359/ree.22-1.15

URL: http://www.una.ac.cr/educare

CORREO: educare@una.cr

La variable género aparece en los totales y en la mayoría de factores. Considerando el género, se vuelve a poner de relieve que las notas se distribuyen de una manera muy similar entre los niños y las niñas, ligeramente superior en las chicas. En Lengua Castellana aparecen unas diferencias algo más visibles, donde las chicas presentan niveles ligeramente más altos (chicas 6.66 y chicos 6.32). Solo aparecen diferencias significativas en Lengua Castellana ( $p=.013)$, lo que hace que no sean significativas para el resto de materias evaluadas.

El curso (edad) es un factor que aparece en tres factores. En rendimiento global las calificaciones se distribuyen de una manera desorganizada. Así, el alumnado de segundo ciclo obtiene los resultados superiores en rendimiento global (6.72), seguido del primero (con una media de 6.51). Las diferencias encontradas fueron significativas para el rendimiento en Lengua Castellana $(p=.003)$, en Conocimiento del Medio $(p=.025)$ y en Inglés $(p=.014)$. No fueron significativas para la media de rendimiento total $(p=.163)$ y el rendimiento en Matemáticas $(p=.087)$.

El estatus aparece en los totales y en un factor que actúa como predictor. Se puede afirmar que el estatus alto es en el que aparecen niveles superiores. En este caso, las medias de cada uno de ellos muestran un nivel algo superior para el estatus medio-alto en el rendimiento global (6.73) y en la mayoría de materias, excepto en Inglés, donde este grupo presenta niveles ligeramente más bajos (6.06). En la media de rendimiento total $(p=.000)$, el rendimiento en Lengua Castellana $(p=.000)$, en Matemáticas $(p=.000)$, en Conocimiento del Medio $(p=.000)$ y en Religión ( $p=.001)$, se encontraron diferencias estadísticamente significativas. De igual manera, en el caso del rendimiento en Inglés $(p=.228)$ no se encontraron.

Para finalizar, se analiza la relación entre el miedo y el rendimiento académico. La influencia parece clara, ambas variables resultan inversamente proporcionales, tal y como recogen los datos presentados en los cálculos regresionales. El grupo que tiene peores resultados (6.05 de media) es el que refleja los niveles más altos de miedo. Una vez fuera de este grupo, la igualdad es mucho más evidente. Quienes reflejan poco (6.31) son los siguientes, seguido de los que manifiestan bastante (6.49). El último (7.05), en cuanto a las calificaciones más bajas, sería el grupo de los que reflejan muy poco donde se registra el menor porcentaje de suspensos (16.1\%). Las diferencias son significativas en el rendimiento en Inglés $(p=.006)$ y el rendimiento global ( $p=.034)$. No ocurre en Lengua $(p=.116)$, Conocimiento del Medio $(p=.107)$, Matemáticas $(p=.107)$ y Religión $(p=.119)$. Se observa que, a pesar de que no se registran diferencias en las materias concretas, salvo en el caso del Inglés, sí que aparecen a nivel de rendimiento total, y disminuyen en los niveles más altos de miedo. 


\section{Discusión y conclusiones}

Para responder al objetivo relacionado con los principales predictores del miedo, se puede afirmar que la población estudiada refleja niveles medio-altos de miedo, donde el factor que genera más miedo es el relacionado con la muerte y menos, el miedo a la crítica y el engaño. El principal predictor de la variable miedo, con el mayor poder determinante en la mayoría de factores es el género. Por tanto, este factor ejerce una influencia clara sobre sus niveles. En todos los casos, las niñas reflejan niveles más altos, algo esperado si se tienen en cuenta los trabajos de Burnham, Hooper y Ogorchock (2011), Cazalla-Luna y Molero (2014) o Pulido y Herrera (2015).

Los patrones de crianza parental son la principal explicación que justifica estas diferencias, en las que el proceso de socialización llevado a cabo por familias y escuelas hace que las chicas sean más proclives a manifestar sus estados emociones de forma libre. Por contra, los chicos aprenden a potenciar su masculinidad desempeñando patrones conductuales en los que se prima la represión de estas emociones, especialmente de aquellas que se consideran más vinculadas al género femenino. Esto es lo que ocurre con el miedo. Esto hace que se siga demandando una mayor igualdad entre géneros, en una intervención más equitativa.

Esta realidad cobra más sentido si se considera el trabajo de Agulló, Filella, Soldevila y Ribes (2011), en el que, en un programa educativo, desarrollado de forma igualitaria, no se encontraron diferencias en las habilidades emocionales; al contrario que ocurre en casos en los que la educación obedece a diferencias en función del género. En condiciones igualitarias, ambos géneros siguen un mismo rimo de aprendizaje. Los niveles inferiores de miedo se hallaron entre los sujetos con estatus más altos (se relacionan con alumnado de cultura cristiana), realidad similar a la encontrada en Miloyan et al. (2014), Merchán et al. (2014) y Pulido y Herrera (2015).

Esta realidad refuerza el hecho de que tanto el estatus, de la misma manera que la cultura, actúan como variables predictoras. En todos ellos se muestra la influencia del entorno sobre el miedo, donde la cultura de origen representa una influencia clara en el desarrollo afectivo. Aquí los patrones aprendidos en el seno familiar inciden en las manifestaciones emocionales del sujeto (Soriano y González, 2013). De esta manera, la intervención educativa que considere la multiculturalidad vuelve a ser una demanda clara, dado que se establecen relaciones directas entre las emociones que puede llegar a desarrollar el alumnado, con independencia de la etapa educativa y el estilo de enseñanza utilizado por cada docente en el desarrollo de su actividad profesional (Rendon, 2015). Existen formas más aptas de orientar las interacciones en el aula de modo adecuado, para que, de esta forma, puedan llegar a facilitar y favorecer una mayor igualdad que haga posible esta educación intercultural.

Relacionado con los predictores del rendimiento académico, la muestra estudiada refleja niveles medio-altos también en rendimiento, con mejores resultados en Religión, y los más bajos en Inglés. En esta ocasión, la cultura es la variable predictora más importante, los grupos 
doi: http://dx.doi.org/10.15359/ree.22-1.15

URL: http://www.una.ac.cr/educare

CORREO: educare@una.cr

musulmanes alcanzan peores resultados en todas las materias, excepto en Inglés. Esta misma realidad se repite en el caso de la variable sociodemográfica estatus. Además de los motivos mencionados en cuanto a las emociones, que vuelven a tener sentido, tiene importancia la lengua materna (Pulido y Herrera, 2015; Roa, 2006) entre ambas etnias, que hacen que aparezcan dificultades para la integración socioeducativa del alumnado no autóctono. El contexto "autóctono" aparece como un importante impulso para el desarrollo socioafectivo. El género actúa también como predictor, a pesar de que la única materia en la que se encontraron diferencias significativas fue en el caso de la materia de Lengua Castellana, donde las chicas alcanzan resultados superiores. La edad también aparece como predictora en varias materias concretas, y es inversamente proporcional al rendimiento.

Para finalizar con la respuesta a los objetivos planteados, se estudió cuál es la relación entre miedo y rendimiento académico. La relación, inversamente proporcional, entre ambas variables parece clara. Existe interacción entre el miedo y el rendimiento total, así como en la mayoría de materias. En el rendimiento total y en la materia de Inglés aparecen además diferencias estadísticamente significativas, aumentan las calificaciones a medida que se desciende en los niveles de miedo. De esta manera, se llega a comprobar que el miedo puede incidir, de manera evidente, en el desempeño académico del sujeto, así como en su interacción con el resto de personas, lo que refuerza lo comentado anteriormente. Por ello, esta emoción adquiere un carácter negativo, por lo que considerar y potenciar las competencias emocionales se antoja una medida necesaria para mejorar el desarrollo evolutivo del sujeto, especialmente entre los grupos más jóvenes (Pulido y Herrera, 2015).

Este es el motivo por el que, tanto la psicología como la educación, han vuelto a interesarse por el mundo emocional. Interés que se manifiesta en la aparición de programas para la intervención socioemocional dirigidos a niños, niñas y adolescentes, cuyo objetivo es mejorar sus competencias emocionales. El modo en el que la persona se relaciona con los demás seres depende de un conjunto de procesos cognitivos, pero también emocionales, procesos que deben ser tenidos en cuenta. Estas competencias emocionales se aprenden interactuando con los demás sujetos. El aprendizaje de este tipo de competencias emocionales suele carecer de un sistema más explícito, de una toma de conciencia y de una reflexión básica (Giménez-Dasí \& Quintanilla, 2009). Por ello, es necesario el fortalecimiento de las habilidades socioemocionales entre el profesorado, para que pueda desempeñar con mayor éxito este tipo de programas. De esta forma, se podrán entrenar habilidades como la percepción, la comprensión y la regulación de las emociones, para mejorar la capacidad de afrontar mejor los eventos laborales estresantes y manejar más adecuadamente las respuestas emocionales negativas (Martín, Berrocal y Brackett, 2008, citados en Chiappe y Cuesta, 2013). De esta manera, se puede crear un ambiente adecuado donde desarrollar este tipo de competencias (Stavroulia, Makri-Botsari, Psycharis y Kekkeris, 2016). 


\section{Referencias}

Agulló, M. J., Filella, G., Soldevila, A. y Ribes, R. (2011). Evaluación de la educación emocional en el ciclo medio de educación primaria. Revista de Educación, 354, 765-783. Recuperado de http://www.revistaeducacion.educacion.es/re354/re354 31.pdf

Ascensio, M., Vila, M. G., Robles-García, R., Páez, F., Fresán, A. y Vázquez, L. (2012). Estudio de traducción, adaptación y evaluación psicométrica del inventario de miedos FSSC-II en una muestra de estudiantes de educación media superior. Salud Mental, 35(3), 195-203. Recuperado de http://www.redalyc.org/articulo.oa?id=58223340003

Balongo, E. y Mérida, R. (2016). El clima de aula en los proyectos de trabajo. Crear ambientes de aprendizaje para incluir la diversidad infantil. Perfiles Educativos, 38(152), 146-162. Recuperado de http://www.redalyc.org/pdf/132/13244824009.pdf

Burnham, J., Hooper, L., \& Ogorchock, H. (2011). Differences in the fears of elementary school children in North and South America: A cross-cultural comparison. International Journal for the Advancement of Counselling, 33(4), 235-251. doi: https://doi.org/10.1007/s10447-011-9131-7

Cazalla-Luna, N. y Molero, D. (2014). Inteligencia emocional percibida, ansiedad y afectos en estudiantes universitarios. Revista Española de Orientación y Psicopedagogía, 25(3), 56-73. doi: https://doi.org/10.5944/reop.vol.25.num.3.2014.13858

Chiappe, A. y Cuesta, J. C. (2013). Fortalecimiento de las habilidades emocionales de los educadores: Interacción en los ambientes virtuales. Educación y Educadores, 16(3), 503524. doi: https://doi.org/10.5294/edu.2013.16.3.6

Extremera, N., Durán, A. y Rey, L. (2009). The moderating effect of trait meta-mood and perceived stress on life satisfaction. Personality and Individual Differences, 47(2), 116-121. doi: https:// doi.org/10.1016/j.paid.2009.02.007

Giménez-Dasí, M. y Quintanilla, L. (2009). “Competencia” social, “competencia” emocional: Una propuesta para intervenir en educación infantil. Infancia y Aprendizaje, 32(3), 359-373. doi: https://doi.org/10.1174/021037009788964222

Gómez-Castro, J. L. (1986). Rendimiento escolar y valores interpersonales: Análisis de resultados en EGB con el cuestionario SIV de Leonardo V. Gordon. Bordón, 262, 257-275.

Gutiérrez, M. y Expósito, J. (2015). Autoconcepto, dificultades interpersonales, habilidades sociales y conductas asertivas en adolescentes. Revista Española de Orientación y Psicopedagogía, 26(2), 42-58. doi: https://doi.org/10.5944/reop.vol.26.num.2.2015.15215

Herrera, F. (2000). La inmigración extranjera no comunitaria y la convivencia en contextos concretos: El caso de Ceuta. En Instituto de Estudios Ceutíes, Monografía de los cursos de Verano de la Universidad de Granada en Ceuta (12a ed., pp. 357-359). Ceuta: Instituto de Estudios Ceutíes - Universidad de Granada. 
Kushnir, J., Gothelf, D., \& Sadeh, A. (2014). Nighttime fears of preschool children: A potential disposition marker for anxiety? Comprehensive Psychiatry, 55(2), 336-341. doi: https://doi. org/10.1016/j.comppsych.2013.08.019

Miloyan, B., Bulley, A., Pachana, N. A., \& Byrne, G. J. (2014). Social phobia symptoms across the adult lifespan. Journal of AffectiveDisorders, 168, 86-90. doi: https://doi.org/10.1016/j. jad.2014.06.029

Merchán, I. M., Bermejo, M. L. y González, J. D. (2014). Eficacia de un programa de educación emocional en educación primaria. Revista de Estudios e Investigación en Psicología y Educación, 1(1), 91-99. doi: https://doi.org/10.17979/reipe.2014.1.1.30

Pena, M., Rey, L., y Extremera, N. (2012). Life Satisfaction and Engagement in Elementary and Primary Educators: Differences in Emotional Intelligence and Gender. Revista de Psicodidáctica, 17(2), 341-358. doi: 10.1387/Rev.Psicodidact.1220

Pinto, B. M. D. C., Dutra, N. B., Filgueiras, A., Juruena, M. F. P. y Stingel, A. M. (2013). Diferenças de gênero entre universitários no reconhecimento de expressões faciais emocionais. Avances en Psicología Latinoamericana, 31(1), 200-222. Recuperado de http://www.redalyc.org/ articulo.oa?id=79928610017

Pulido, F. y Herrera, F. (2015). Miedo e inteligencia emocional en el contexto pluricultural de Ceuta. Anuario de Psicología, 45(2), 249-263. Recuperado de http://www.redalyc.org/ articulo.oa?id $=97044007008$

Rendon, M. A. (2015). Education of social and emotional competence and teaching styles in high school. ¿OФIA-Sophia, 11(2), 237-256. Recuperado de http://revistas.ugca.edu.co/ index.php/sophia/article/viewFile/353/804

Roa, J. M. (2006). Rendimiento escolar y "situación diglósica" en una muestra de escolares de educación primaria en Ceuta. Revista Electrónica de Investigación Educativa, 8(1), 1-15. Recuperado de https://redie.uabc.mx/redie/article/view/124/215

Soriano, E. y González, A. J. (2013). Las competencias emocionales en el ámbito afectivo-sexual de los adolescentes autóctonos e inmigrantes. Revista de Investigación Educativa, 31(1), 133-149. doi: https://doi.org/10.6018/rie.31.1.150531

SPSS. (2009). PASW Statistics for Windows, Version 18.0. Chicago, Il: Autor.

Stavroulia, K. E., Makri-Botsari, E., Psycharis, S., \& Kekkeris, G. (2016). Emotional experiences in simulated classroom training environments. International Journal of Information and Learning Technology, 33(3), 172-185. doi: https://doi.org/10.1108/IJILT-10-2015-0030 